\title{
Brown preadipocyte transplantation locally ameliorates obesity
}

\author{
Kento Takaya ${ }^{1}$, Naruhito Matsuda ${ }^{1}$, Toru Asou ${ }^{2}$, Kazuo Kishi ${ }^{1}$ \\ ${ }^{1}$ Department of Plastic and Reconstructive Surgery, Keio University School of Medicine, Tokyo; ${ }^{2}$ Tokyo Cosmetic Surgery Clinic, Tokyo, Japan
}

Background Brown adipose tissue (BAT) is a potential target for anti-obesity treatments. Previous studies have shown that BAT activation causes an acute metabolic boost and reduces adiposity. Furthermore, BAT and BAT-derived cell transplantation reportedly help treat obesity by regulating glucose and fatty acid metabolism. However, since BAT transplantation leads to whole-body weight loss, we speculated that earlier approaches cause a generalized and unnecessary fat tissue loss, including in breast and hip tissues.

Methods We transplanted white adipose tissue-derived or BAT-derived preadipocytes prepared from C57BL/6 mice into one side of the inguinal fat pads of an obese mouse model $(\mathrm{db} /$ $d b$ mice) to examine whether it would cause fat loss at the peri-transplant site ( $\mathrm{n}=5$ each). The same volume of phosphate-buffered saline was injected as a control on the other side. Six weeks after transplantation, the inguinal fat pad was excised and weighed. We also measured the concentrations of glucose, triglycerides, fatty acids, and total cholesterol in the peripheral blood.

Results BAT-derived preadipocytes showed abundant mitochondria and high levels of mitochondrial membrane uncoupling protein 1 expression, both in vivo and in vitro, with a remarkable reduction in weight of the inguinal fat pad after transplantation $(0.17 \pm 0.12 \mathrm{~g}$, $P=0.043)$. Only free fatty acid levels tended to decrease in the BAT-transplanted group, but the difference was not significant $(\mathrm{P}=0.11)$.

Conclusions Our results suggest that brown adipocytes drive fat degradation around the transplantation site. Thus, local transplantation of BAT-derived preadipocytes may be useful for treating obesity, as well as in cosmetic treatments.

Keywords Adipose tissue, brown / Adipocytes / Transplantation / Weight loss / Obesity

\author{
Correspondence: Kento Takaya \\ Department of Plastic and \\ Reconstructive Surgery, Keio \\ University School of Medicine, 35 \\ Shinanomachi, Shinjuku-ku, Tokyo \\ 160-8582, Japan \\ Tel: +81-3-5363-3814 \\ Fax: +81-3-3352-1054 \\ E-mail: kento-takaya312@keio.jp
}

This work was supported by Japan Society for the Promotion of Science (JSPS) KAKENHI (grant No. JP12877288).

Received: November 18, $2020 \bullet$ Revised: April 15, $2021 \bullet$ Accepted: May 4, 2021

pISSN: 2234-6163 • elSSN: 2234-6171 • https://doi.org/10.5999/aps.2020.02257 • Arch Plast Surg 2021;48:440-447

\section{INTRODUCTION}

The incidence of obesity is increasing at a tremendous rate. Currently, over $50 \%$ of the world's population is classified as overweight [1]. Obesity is caused by an imbalance between energy intake and energy expenditure. Adipose tissue is an important organ involved in maintaining metabolic function throughout the body. Obesity is characterized by an increased risk of comorbidities, such as type 2 diabetes and cardiovascular disease $[2,3]$.

Thus, there is a growing demand for drugs both to counter obesity and to enhance body image. However, anti-obesity drugs generally cause whole-body weight loss, rather than targeting specific areas. Of note, systemic weight loss might not 
align well with the aesthetic requirements to maintain adequate adipose tissue on desired areas, such as the breast and hips.

Surgical resection and liposuction are used to reduce local fat; however, these procedures create new wounds in the patient and carry the risk of hematomas, seromas, and infections [4]. Local injections of amino acids and steroids, known as mesotherapy, are also effective; however, they cause side effects, such as iatrogenic Cushing disease in the case of steroids [5]. Therefore, it would be desirable to develop a method that would be both safe and effective for reducing local fat.

Both rodents and humans have two types of adipose tissue. The first is white adipose tissue (WAT), which makes up nearly all adipose tissue, is found in the body's subcutaneous fat and visceral fat. WAT is characterized by large, monolocular lipid droplets that occupy most of the intracellular space, and it acts as a triglyceride reservoir to steadily release energy during a fast or physical activity [6]. The second type is known as brown adipose tissue (BAT), which forms small multilocular lipid droplets, unlike WAT. BAT is present in each scapula, the upper jaw, and the kidney, and contains abundant mitochondria. BAT employs mitochondrial membrane uncoupling protein 1 (UCP-1) to metabolize glucose or free fatty acids to fuel triglyceride catabolism $[7,8]$. BAT cell activity is thought to be induced by cold stimulation [9], norepinephrine [10], and sympathetic stimulation [11]. Adult humans contain activated BAT, the activity of which is thought to decrease with age, obesity, or progression of diabetes. BAT shows potential for treating obesity [12]. Several previous studies in laboratory animals showed that BAT could dissipate heat, thereby reducing both obesity and insulin sensitivity $[13,14]$. Maintaining BAT in an active state to constantly burn excess food energy may be a new approach for treating obesity and obesity-related disorders.

Several studies on BAT and BAT preadipocyte transplantation have been performed since the 1960s. Reportedly, BAT transplantation helps to reduce body weight and improve glucose metabolism and insulin resistance [15-17]. Of note, subcutaneous, intraperitoneal, and epidermal WAT have been used as ideal transplantation sites. However, to the best of our knowledge, no reports have described local fat loss limited to the transplantation site.

In this study, we examined whether local fat loss could be achieved via the transplantation of cultured BAT preadipocytes.

\section{METHODS}

The study protocol was reviewed and approved by the Institutional Animal Care and Use Committee of Keio University School of Medicine (IACUC No. 13072-(2)). All experiments were performed in accordance with our Institutional Guidelines on Animal Experimentation.

\section{Culture of WAT and BAT cells}

Five C57BL/6J mice (6 weeks old) were used to obtain WAT and BAT cells. All the mice were obtained from Sankyo Labo Service Corporation, Inc. (Tokyo, Japan). After sacrificing the mice by cervical dislocation, the skin was dissected with scissors. WAT samples were aseptically dissected from the inguinal lesion, and BATs samples were obtained from connective tissue between the scapulae. The BAT was easily identified by its characteristic brown color (Fig. 1). Each tissue was fixed with 10\% formalin. After mincing the dissected tissues with a surgical knife, they were incubated in $0.2 \%$ collagenase (Godo Shusei, Tokyo, Japan) for 30 minutes at $37^{\circ} \mathrm{C}$ with pipetting every 5 minutes. After digestion with collagenase, the cells were collected by centrifugation at $200 \times g$ for 5 minutes. The cells were cultured in Dulbecco's modified Eagle's medium (DMEM; Wako Pure Chemical Industries, Osaka, Japan) containing 10\% fetal bovine serum (Thermo Fisher Scientific, Waltham, MA, USA) and $1 \%$ penicillin/streptomycin (Thermo Fisher Scientific). The medium was changed twice a week. To avoid differentiation into mature adipocytes, newborn calf serum (NBCS) and insulin were not used. Subculturing was performed with $0.25 \%$ trypsin/EDTA (Sigma-Aldrich, St. Louis, MO, USA). Cells from passages 3 to 5 were used for analysis.

The cultured preadipocytes were evaluated based on their ability to differentiate into mature adipocytes by changing the medium into DMEM containing 10\% NBCS (Thermo Fisher Scientific), $1 \%$ penicillin/streptomycin, and $0.5 \mu \mathrm{g} / \mathrm{mL}$ insulin (Wako Pure Chemical Industries). The characteristics of the BAT were evaluated by mitochondrial staining with Rh123
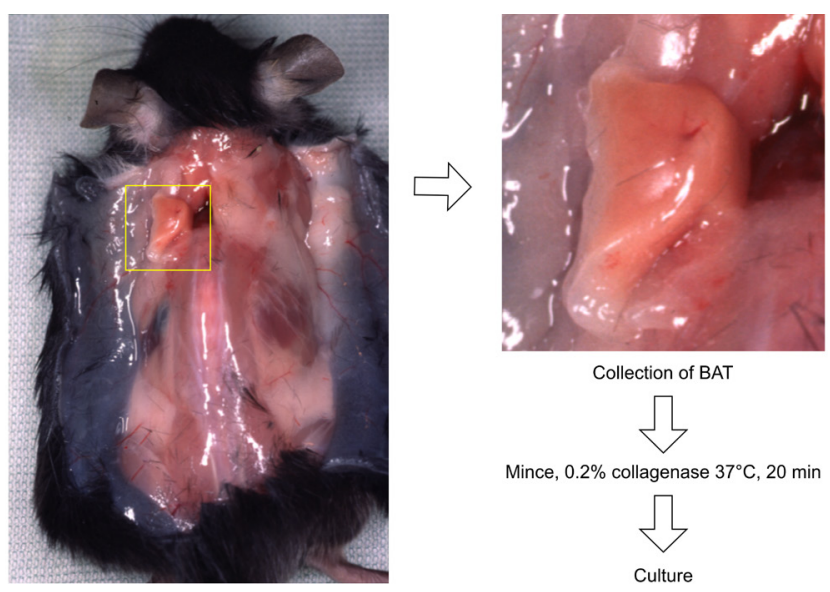

Fig. 1. Processing brown adipose tissue (BAT) around the scapula. A midline incision in the back reveals adipose tissue at the base of the arm. This was collected as axillary BAT. 
(Dojindo, Kumamoto, Japan) and immunostaining using a polyclonal anti-UCP-1 antibody (Santa Cruz Biotechnology, Dallas, TX, USA). The cells were placed on glass slides, fixed with acetone for 5 minutes at room temperature $\left(15^{\circ} \mathrm{C}-25^{\circ} \mathrm{C}\right)$, and dried completely before staining. The samples were incubated with primary antibody diluted to 1:100 with phosphatebuffered saline (PBS) for 60 minutes at room temperature. After washing 3 times with PBS, biotin-conjugated goat anti-mouse IgG (Vector Laboratories, Burlingame, CA, USA) was used as the secondary antibody, which was incubated with the cells for 30 minutes at room temperature. After washing 3 times with PBS, Streptavidin Alexa Fluor 488 Conjugate (Life Technologies, Carlsbad, CA, USA) in PBS (1:100) was added to the samples and incubated for another 30 minutes. Counterstaining of the nucleolus was performed with 1:500 Hoechst 33342 (Life Technologies) for 15 minutes.

\section{Transplantation of cultured preadipocytes}

Eight-week-old male $\mathrm{C} 57 \mathrm{BL} / \mathrm{db} / \mathrm{db}$ mice were used as recipients of the cultured cells because they showed increased levels of fat tissue following overeating (Fig. 2). Five mice each were used for transplantation of WAT-derived and BAT-derived preadipocytes. Cells from passages 3-5 were collected by trypsinization. After centrifugation, the cells were stained with $0.25 \%$ DiI (Life Technologies) for 5 minutes on ice and washed with PBS. Cells $\left(1 \times 10^{7}\right)$ suspended in $0.5 \mathrm{~mL}$ of PBS were transplanted using a 27-gauge insulin syringe into one side of the inguinal fat pad of the $d b / d b$ mice. As a control, the opposite side of the fat pad was injected with the same volume of PBS without cells. Six weeks after the transplantation, the mice were sacrificed by cervical dislocation, and the skin was dissected using scissors. Transplanted cells were collected and examined by fluorescence analysis of DiI. After dissection, the weight of the dissected fat pad was immediately determined using a microbal-

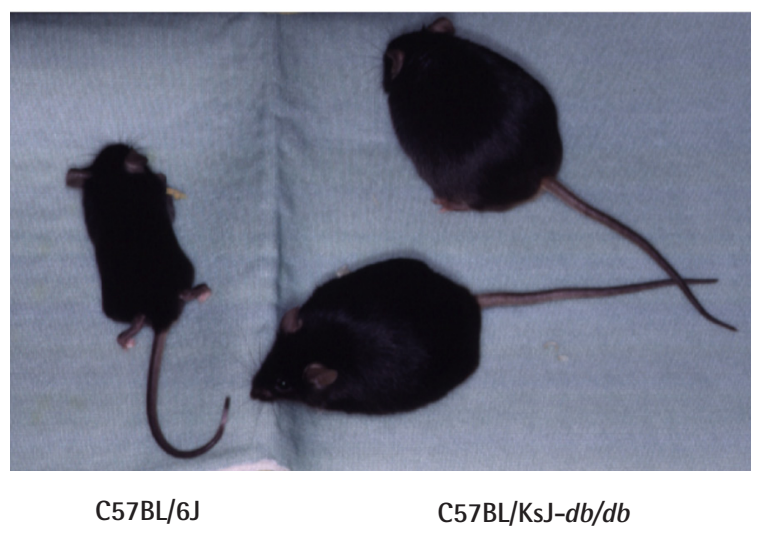

Fig. 2. Difference between $\mathrm{C} 57 \mathrm{BL} / 6 \mathrm{~J}$ and $\mathrm{C} 57 \mathrm{BL} / \mathrm{KsJ}-d b / d b$ mice. $\mathrm{C} 57 \mathrm{BL} / \mathrm{KsJ}-d b / d b$ mice have a larger body and are obese. ance. The fat pad is defined as the adipose tissue surrounded by a membrane located inside the thigh muscle, and it was carefully excised without including the subcutaneous fat or muscle. Just after cervical dislocation, thoracotomy was performed to collect peripheral blood from the heart. Blood glucose, free fatty acid, triglyceride, and total cholesterol levels in the peripheral blood were examined.

\section{Statistical analysis}

The statistical significance of differences between treatment groups was determined using Statistica software version 9.0 (StatSoft, Inc., Tulsa, OK, USA). Differences were considered significant at $\mathrm{P}<0.05$. Single end-point measures were compared using the paired-sample t-test.

\section{RESULTS}

An evaluation of histological sections revealed that the brown tissue collected between the scapulae and the white tissue from the inguinal lesion almost entirely consisted of adipose tissue. Additionally, WAT and BAT did not show a mixed pattern. The size of the intracellular fat droplets was 2- to 3-fold larger in WAT than in BAT, as reported previously (Fig. 3). Cryosections of each tissue indicated that the cytoplasm of the BAT was rich

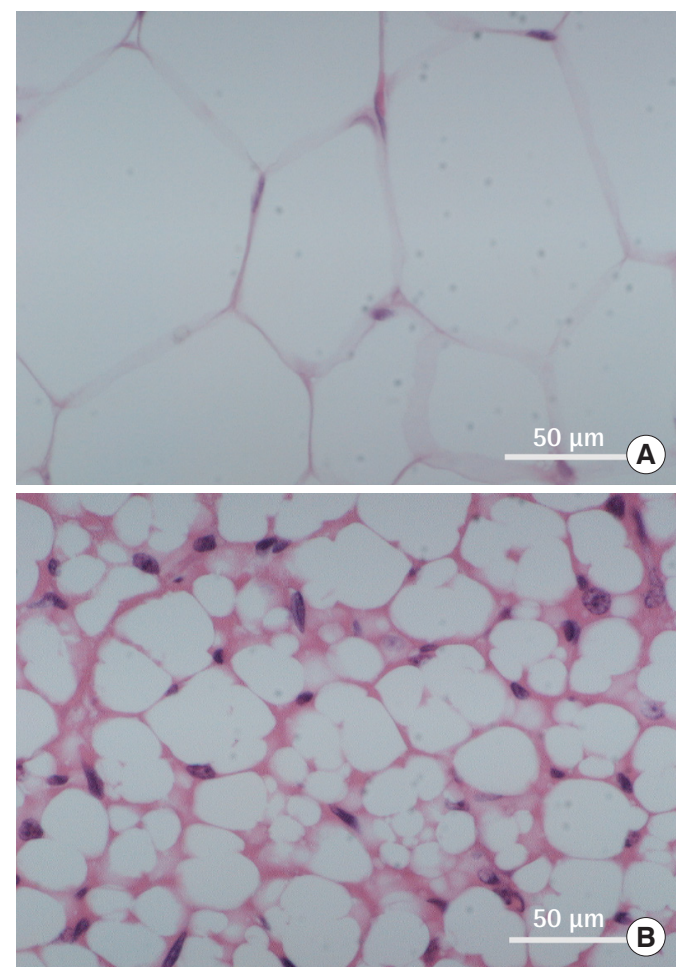

Fig. 3. Histological sections. (A) White adipose tissue: large, monolobular lipid droplets (H\&E). (B) Brown adipose tissue: small multilocular lipid droplets (H\&E). 
in mitochondria (Fig. 4). Cells were successfully cultured in growth medium. Approximately $90 \%$ of the cultured cells from BAT exhibited high cytoplasmic UCP-1 expression after culture, whereas very low UCP-1 expression was observed in cells obtained from WAT (Fig. 5). Therefore, cultured BAT cells were rich in mitochondria. We confirmed that the WAT and BAT preadipocytes labeled with DiI were successfully engrafted onto the fat pad in the inguinal region of $d b / d b$ mice (Fig. 6).

Transplantation of WAT cells into the inguinal fat pads of $d b /$ $d b$ mice significantly increased the weight of the fat pads by $5 \%$ to $10 \%$, when compared to the weight of the fat pad on the other side $(\mathrm{P}=0.001)$. The weight of the BAT-implanted fat pads was significantly reduced by $5 \%$ to $30 \%$, when compared to the weight of the control $(\mathrm{P}=0.043)$ (Fig. 7).

Although blood sugar glucose, triglyceride, and total cholesterol levels in the peripheral blood showed no significant differences between the WAT-transplanted group and BAT-transplanted group, free fatty acid levels showed a tendency to be lower in the BAT-transplanted group (Fig. 8). However, no statistically significant difference was observed $(\mathrm{P}=0.11)$.
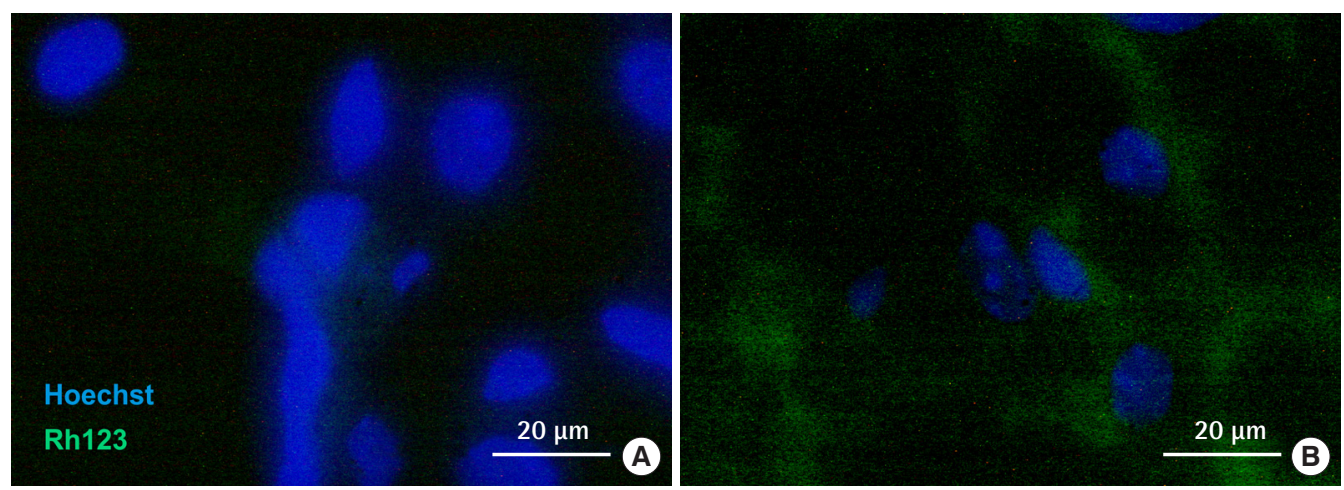

Fig. 4. Fluorescence immunostaining of mitochondria in white adipose tissue (A) and brown adipose tissue (B). Abundant mitochondria stained with Rh123 were observed in the cytoplasm of brown adipose tissue preadipocytes.

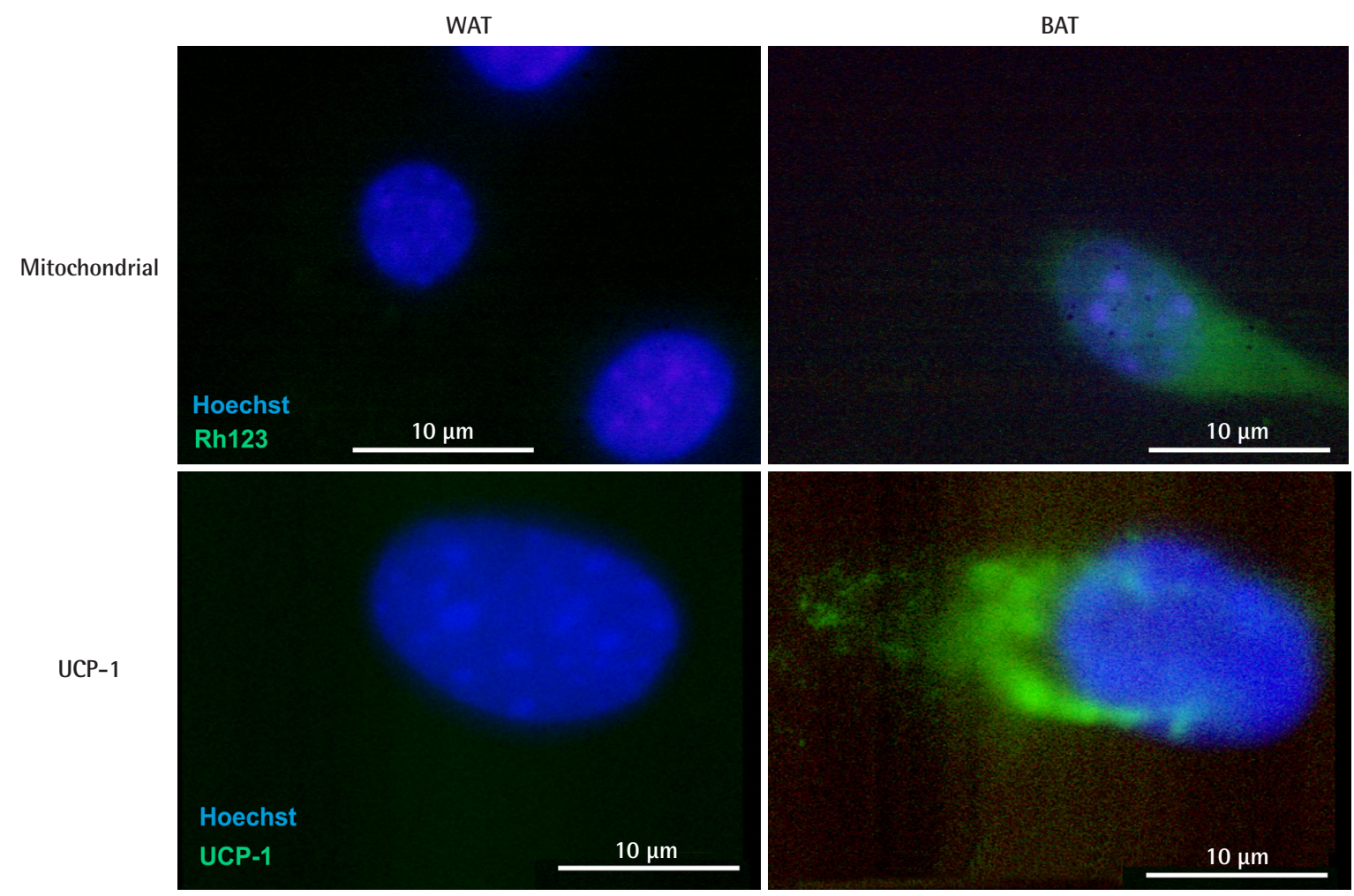

Fig. 5. Fluorescence immunostaining of mitochondria and UCP-1 in preadipocytes. Abundant mitochondria were observed in BAT preadipocytes as well as in BAT. Specific expression of UCP-1 was observed in the cytoplasm of BAT preadipocytes. UCP-1, uncoupling protein 1; BAT, brown adipose tissue; WAT, white adipose tissue. 

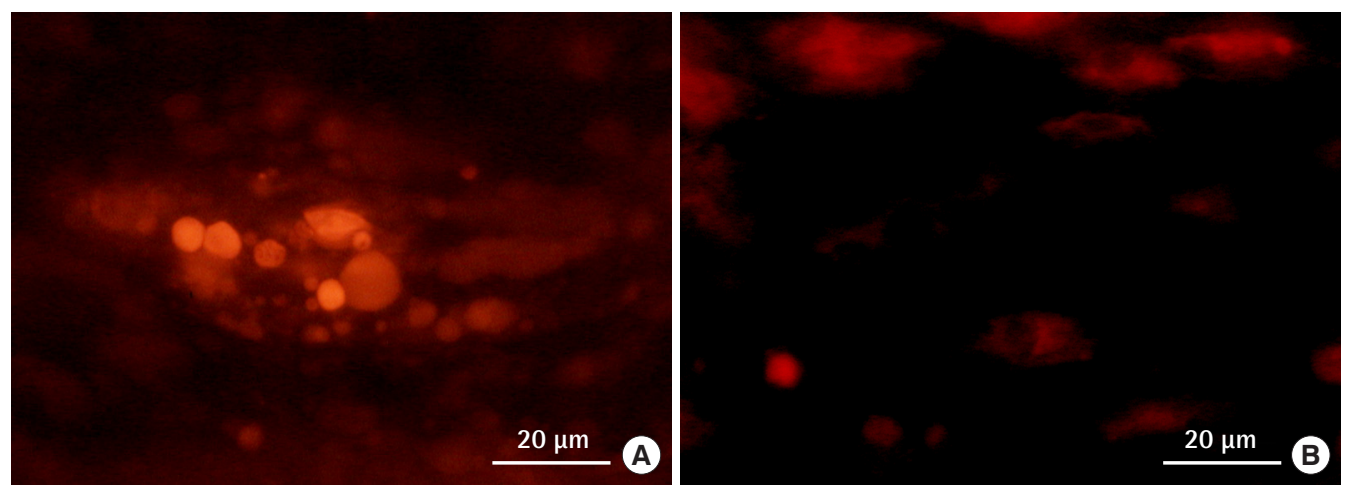

Fig. 6. Brown adipose tissue preadipocytes transplanted into an inguinal fat pad. The transplanted cells were labeled with Dil, and engraftment in the tissue was observed. (A) White adipose tissue-preadipocytes. (B) Brown adipose tissue-preadipocytes.
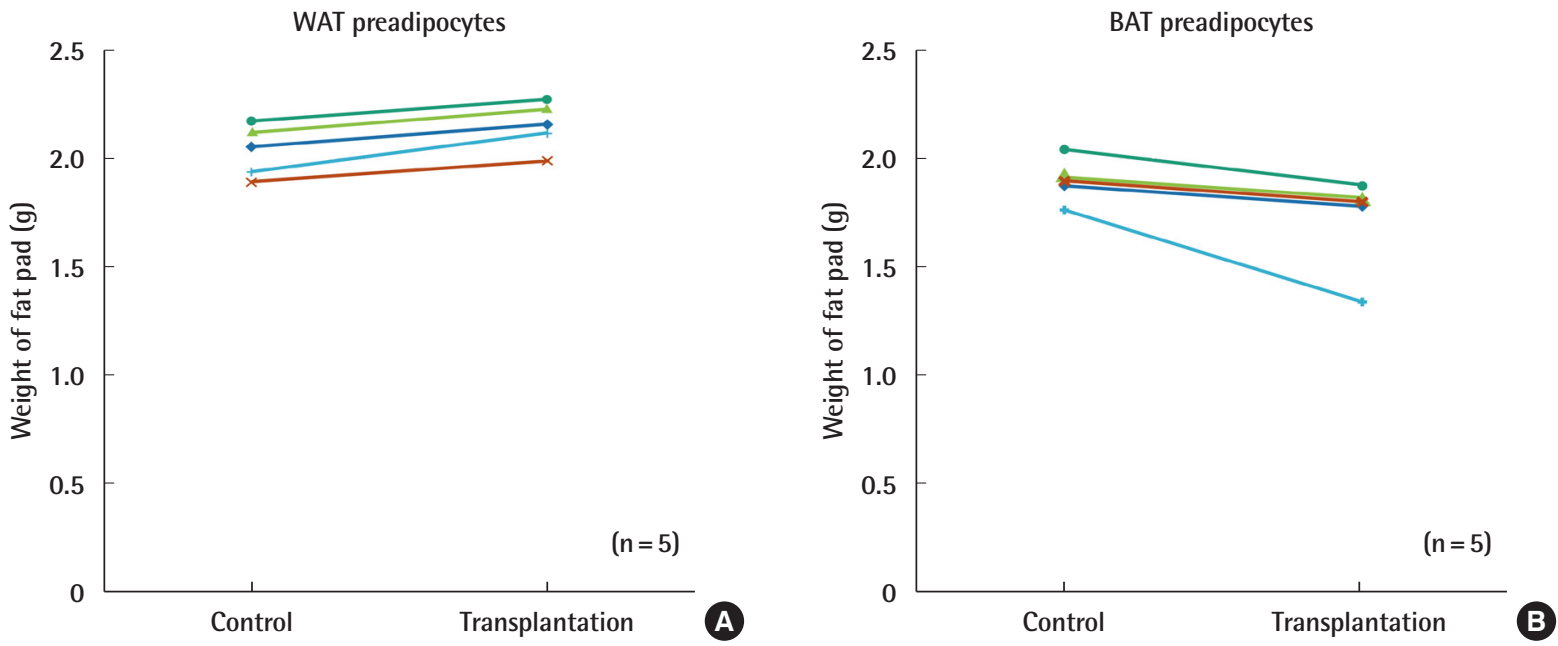

Fig. 7. Changes in the weight of fat pads following transplantation. (A) Comparison between WAT preadipocytes and control (PBS). (B) Comparison between BAT preadipocytes and control (PBS). All BAT-transplanted groups showed decreased fat weight. WAT, white adipose tissue; BAT, brown adipose tissue; PBS, phosphate-buffered saline.

\section{DISCUSSION}

BAT is a potential therapeutic target for ameliorating obesity and its associated comorbidities because of its inherent thermogenic potential and ability to improve glucose metabolism. BAT activation, either by pharmacological treatment or by cold exposure, acutely increases metabolic function and reduces adiposity [16-18]. Furthermore, in a transplant model, BAT activation reportedly improved glucose metabolism and metabolic health [19]. Although previous studies assessed systemic weight loss, measuring the whole-body weight of the implanted animal does not provide information related to local fat loss. In addition, the weight of an animal might vary slightly throughout the day. Additionally, although fat is systemically reduced, changes in the appearance and morphology of obese animal models have not been analyzed. In this study, we investigated whether local transplantation of BAT-derived cultured preadipocytes causes local fat loss in the peri-transplant tissue. As the inguinal fat pad is present along the margin of the iliac bone and isolated from surrounding fat tissues by loose areolar fascial tissue, we targeted this area to enable precise and localized weight loss comparisons.

BAT energy expenditure depends on the presence of abundant mitochondria in the cytoplasm and UCP-1 on the mitochondrial surface. It has been reported that stimulating the activity of UCP-1 in brown adipocytes increases energy expenditure. Brown adipocytes treated with the beta-3 adrenergic agonist CL 316,243 showed a significantly increased resting metabolic rate [20]. A previous study showed that the energy expenditure of brown adipocytes is regulated directly or via beta- 3 adrenergic receptor agonists [21]. UCP-1 expression positively correlates with metabolic inefficiency and increases with cold acclimation and supercharging, and BAT transplantation reduces both diet-induced and genetic obesity [22]. Nerve growth factor, synthesized and released by brown adipocytes, regulates 

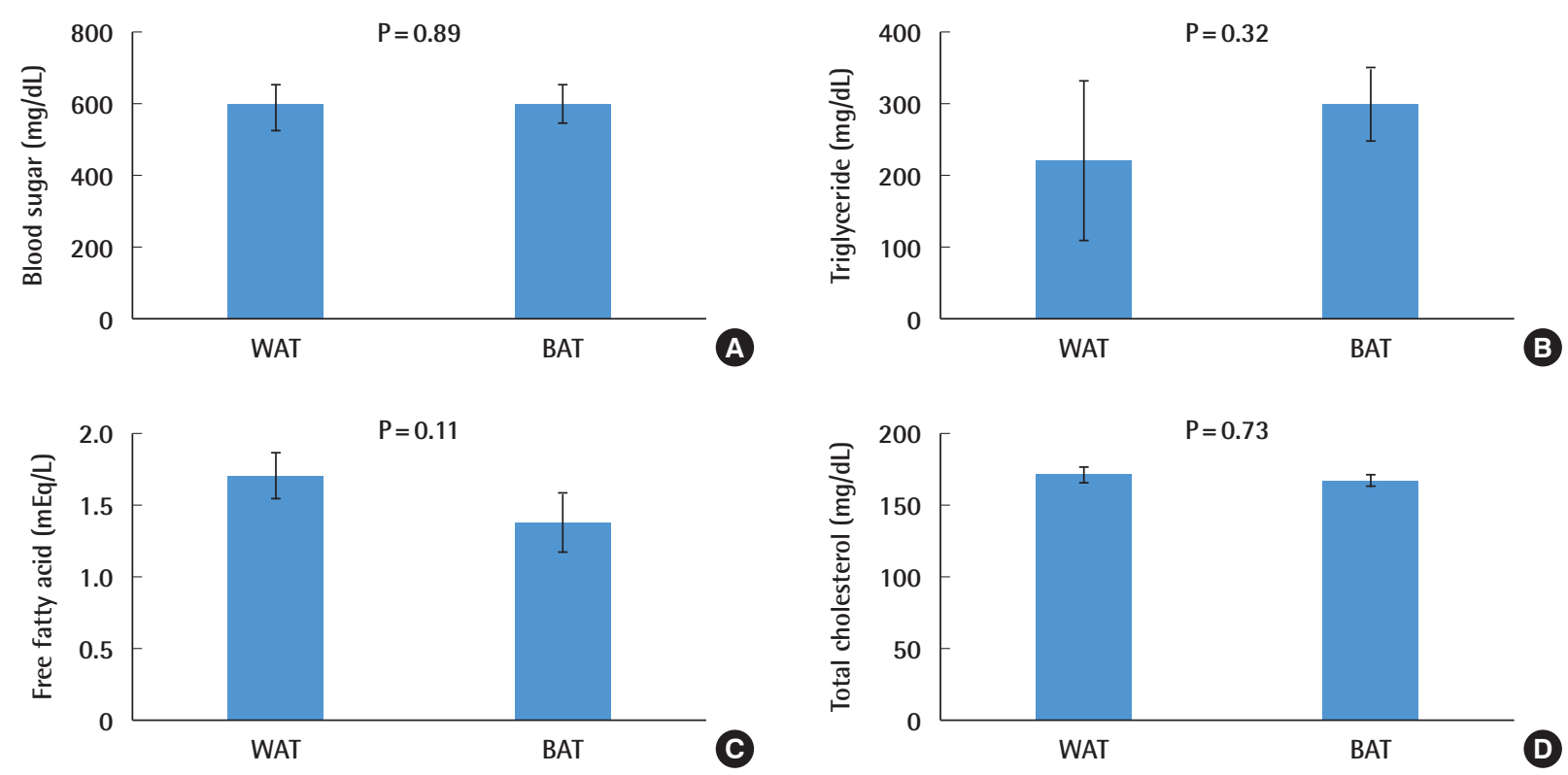

Fig. 8. Changes in peripheral blood parameters following transplantation. (A) Blood sugar. (B) Triglycerides. (C) Free fatty acids. (D) Total cholesterol. Decreased levels of free fatty acids were observed in the BAT preadipocyte transplantation group. Other parameters did not show a statistically significant difference. WAT, white adipose tissue; BAT, brown adipose tissue.

obesity [23]. The expression of UCP-1 in adipocytes is regulated by the fat-specific promoter of $a P 2-U c p 1$, which, in transgenic mice, attenuates obesity induced by genetic or dietary factors [24]. These results indicate that increasing UCP-1 expression in mitochondria might be one of the most successful strategies to recalibrate energy consumption.

In previous studies, BAT transplantation improved glucose levels and increased insulin sensitivity while reducing inflammation [19]. These results were accompanied by increased levels of certain hormones, such as adiponectin and norepinephrine, resulting in higher metabolic rates, oxygen consumption, and energy expenditure [25]. Additionally, BAT preadipocytes proliferate in response to pro-angiogenic factors responsible for the expansion of the capillary network. BAT-derived adipocytes begin expressing UCP-1 in response to the activation of adenylate cyclase [26]. Adipocytes express neuroendocrine and secretory factors, including the proprotein convertase PCSK1, which is strongly associated with obesity [27]. Activated beige/bright adipocytes affect systemic glucose homeostasis, potentially via a neuroendocrine mechanism.

This study demonstrated an abundant mitochondrial population and robust UCP-1 expression in cultured brown preadipocytes. These results indicate that even cells cultured from BAT may consume energy, possibly through an endocrine mechanism, following transplantation of cultured brown preadipocytes.

We also found that the transplantation of brown preadipocytes reduced the weight of the transplanted inguinal fat pads. Previ- ous studies of BAT and BAT-derived cell transplantation targeted subcutaneous fat, visceral fat, areas under the kidney capsule, and intraperitoneal regions as transplant sites, and improved glucose homeostasis and insulin sensitivity, resulting in systemic fat loss. However, the fat pad in the groin is anatomically isolated by fascia. There are also reports that transplantation onto the groin fat pad shows a higher graft retention rate than subcutaneous or intramuscular transplantation in mice [28]. Thus, it is possible that the BAT-derived preadipocytes transplanted into the fat pad of the groin localized to the fat pad and caused a local decrease in fat by an endocrine mechanism via adipokines.

Peripheral blood data also showed that blood glucose, triglyceride, total cholesterol, and free fatty acid levels did not change significantly after brown preadipocyte transplantation. Unlike previous studies on BAT and BAT preadipocyte transplantation, our results suggest that local glucose metabolism occurs following the transplantation of brown preadipocytes.

This study has a few limitations. First, only for the fat pad in the groin area was weighed, and effects on other murine adipose tissues were not monitored. Second, the long-term course after cell transplantation is unknown. However, it has been reported that the UCP-1 expression of transplanted BAT can be maintained only for about 2 months after transplantation [29]. Therefore, long-term studies will be needed to analyze the relationship between increased energy consumption from heat production and compensatory mechanisms, such as increased appetite, that may overcome the benefits of transplantation. Third, the safety of BAT and BAT-derived cell transplantation was not 
evaluated. To date, no obvious adverse events have been reported in mouse transplant models, but it would be necessary to consider potential complications, such as abnormal hyperthermia, necrosis, tachycardia, and palpitations due to hyperthermia, when considering future human applications. To overcome these limitations, in future studies, we plan to perform cell transplantation in other tissues and observe long-term changes in the weight of adipose tissue.

In conclusion, preadipocytes cultured from BAT contained abundant mitochondria and expressed UCP-1. BAT-derived preadipocytes cultured in vitro were assumed to mimic in vivo metabolism. Based on these results, transplantation of cultured brown preadipocytes may be suitable as a local fat reduction strategy to treat obesity and attain patients' aesthetic goals.

\section{NOTES}

\section{Conflict of interest}

No potential conflict of interest relevant to this article was reported.

\section{Ethical approval}

The study protocol was reviewed and approved by the Institutional Animal Care and Use Committee of Keio University School of Medicine (IACUC No. 13072-(2)). All experiments were performed in accordance with our Institutional Guidelines on Animal Experimentation.

\section{Author contribution}

Conceptualization: K Takaya, N Matsuda, T Asou, K Kishi. Methodology: K Takaya, K Kishi. Validation, formal analysis, data curation, visualization: K Takaya, N Matsuda. Supervision, project administration: T Asou, K Kishi. Funding acquisition: $\mathrm{K}$ Kishi. Writing - original draft: K Takaya. Writing - review \& editing: T Asou, K Kishi.

\section{ORCID}

$\begin{array}{ll}\text { Kento Takaya } & \text { https://orcid.org/0000-0001-8992-2762 } \\ \text { Naruhito Matsuda } & \text { https://orcid.org/0000-0001-6671-969X } \\ \text { Toru Asou } & \text { https://orcid.org/0000-0001-9312-3584 } \\ \text { Kazuo Kishi } & \text { https://orcid.org/0000-0002-4298-9828 }\end{array}$

\section{REFERENCES}

1. World Health Organization (WHO). Obesity and overweight [Internet]. Geneva: WHO; c2017 [cited 2020 Oct 30]. Available from: https://www.who.int/news-room/ fact-sheets/detail/obesity-and-overweight.
2. Thomou T, Mori MA, Dreyfuss JM, et al. Adipose-derived circulating miRNAs regulate gene expression in other tissues. Nature 2017;542:450-5.

3. Grundy SM, Adams-Huet B, Vega GL. Variable contributions of fat content and distribution to metabolic syndrome risk factors. Metab Syndr Relat Disord 2008;6:281-8.

4. Shiffman MA. Prevention and treatment of liposuction complications. In: Shiffman MA, Di Giuseppe A, editors. Liposuction-principles and practice. 1st ed. New York: Springer; 2006. p. 333-41.

5. Jayasinghe $S$, Guillot T, Bissoon L, et al. Mesotherapy for local fat reduction. Obes Rev 2013;14:780-91.

6. Frayn KN. Fat as a fuel: emerging understanding of the adipose tissue-skeletal muscle axis. Acta Physiol (Oxf) 2010; 199:509-18.

7. Heaton GM, Wagenvoord RJ, Kemp A Jr, et al. Brown-adipose-tissue mitochondria: photoaffinity labelling of the regulatory site of energy dissipation. Eur J Biochem 1978;82: 515-21.

8. Bartelt A, Bruns OT, Reimer R, et al. Brown adipose tissue activity controls triglyceride clearance. Nat Med 2011;17: 200-5.

9. van Marken Lichtenbelt WD, Vanhommerig JW, Smulders $\mathrm{NM}$, et al. Cold-activated brown adipose tissue in healthy men. N Engl J Med 2009;360:1500-8.

10. Collins S, Surwit RS. The beta-adrenergic receptors and the control of adipose tissue metabolism and thermogenesis. Recent Prog Horm Res 2001;56:309-28.

11. Cannon B, Nedergaard J. Brown adipose tissue: function and physiological significance. Physiol Rev 2004;84:277359.

12. Zingaretti MC, Crosta F, Vitali A, et al. The presence of UCP1 demonstrates that metabolically active adipose tissue in the neck of adult humans truly represents brown adipose tissue. FASEB J 2009;23:3113-20.

13. Zhang Y, Xu Q, Liu YH, et al. Medium-chain triglyceride activated brown adipose tissue and induced reduction of fat mass in C57BL/6J mice fed high-fat diet. Biomed Environ Sci 2015;28:97-104.

14. Virtanen KA, Lidell ME, Orava J, et al. Functional brown adipose tissue in healthy adults. N Engl J Med 2009;360: 1518-25.

15. Silva FJ, Holt DJ, Vargas V, et al. Metabolically active human brown adipose tissue derived stem cells. Stem Cells 2014; 32:572-81.

16. Zhu Z, Spicer EG, Gavini CK, et al. Enhanced sympathetic activity in mice with brown adipose tissue transplantation (transBATation). Physiol Behav 2014;125:21-9. 
17. Stanford KI, Middelbeek RJ, Townsend KL, et al. Brown adipose tissue regulates glucose homeostasis and insulin sensitivity. J Clin Invest 2013;123:215-23.

18. Misra A, Vikram NK. Clinical and pathophysiological consequences of abdominal adiposity and abdominal adipose tissue depots. Nutrition 2003;19:457-66.

19. Liu X, Wang S, You Y, et al. Brown adipose tissue transplantation reverses obesity in $\mathrm{Ob} / \mathrm{Ob}$ mice. Endocrinology 2015;156:2461-9.

20. Ghorbani M, Teimourian S, Farzad R, et al. Apparent histological changes of adipocytes after treatment with $\mathrm{CL}$ 316,243, a $\beta$-3-adrenergic receptor agonist. Drug Des Devel Ther 2015;9:669-76.

21. Harper JA, Dickinson K, Brand MD. Mitochondrial uncoupling as a target for drug development for the treatment of obesity. Obes Rev 2001;2:255-65.

22. Symonds ME, Aldiss P, Dellschaft N, et al. Brown adipose tissue development and function and its impact on reproduction. J Endocrinol 2018;238:R53-62.

23. Sornelli F, Fiore M, Chaldakov GN, et al. Adipose tissue-derived nerve growth factor and brain-derived neurotrophic factor: results from experimental stress and diabetes. Gen
Physiol Biophys 2009;28 Spec No:179-83.

24. Kopecky J, Flachs P, Bardova K, et al. Modulation of lipid metabolism by energy status of adipocytes: implications for insulin sensitivity. Ann N Y Acad Sci 2002;967:88-101.

25. Kikai M, Yamada H, Wakana N, et al. Adrenergic receptormediated activation of FGF-21-adiponectin axis exerts atheroprotective effects in brown adipose tissue-transplanted apoE -/- mice. Biochem Biophys Res Commun 2018;497: 1097-103.

26. Min SY, Kady J, Nam M, et al. Human 'brite/beige' adipocytes develop from capillary networks, and their implantation improves metabolic homeostasis in mice. Nat Med 2016;22:312-8.

27. Muhsin NIA, Bentley L, Bai Y, et al. A novel mutation in the mouse Pcsk1 gene showing obesity and diabetes. Mamm Genome 2020;31:17-29.

28. Shi Y, Yuan Y, Dong Z, et al. The fate of fat grafts in different recipient areas: subcutaneous plane, fat pad, and muscle. Dermatol Surg 2016;42:535-42.

29. Gunawardana SC, Piston DW. Reversal of type 1 diabetes in mice by brown adipose tissue transplant. Diabetes 2012;61: 674-82. 